\title{
二乙基锌对原位产生吲哚亚胺的对映选择性加成反应
}

\author{
倪承燕李文科何 龙* 刘全忠* 康泰然
}

$\left({ }^{a}\right.$ 西华师范大学化学化工学院 化学合成与污染控制四川省重点实验室 南充 637000)

\begin{abstract}
摘要 发展了乙基锌对原位产生的不饱和亚胺的对映选择性共轭加成, 磺酰吲哚衍生物在乙基锌存在下原位生成不饱 和亚胺，手性亚磷酰胺配体一铜络合物催化二乙基锌对该原位产生的不饱和亚胺反应以最高 99\%的产率和 $80 \%$ 的对映 选择性得到了一系列 3 位取代的光学活性的吲哚衍生物.
\end{abstract}

关键词＼cjkstart对映选择性共轭加成; 磺酰吲哚衍生物; 不饱和亚胺

\section{Enantioselective Conjugate Addition of Diethylzinc to Vinylogous Imines Generated in situ from Sulfonyl Indoles}

\author{
$\mathrm{Ni}$, Chengyan Li, Wenke He, Long* Liu, Quanzhong* Kang, Tairan \\ (Chemical Synthesis and pollution Control Key Laboratory of Sichuan Province, College of Chemistry and \\ Chemical Engineering, China West Normal University, Nanchong 637000)
}

\begin{abstract}
Enantioselective conjugate addition of diethyl zinc to vinylogous imines generated in situ from sulfonyl indoles was developed. Various sulfonyl indoles reacted with diethyl zinc in the presence of chiral phosphoramidites in combination with copper triflate affording the corresponding indole substituted alkanes in up to $99 \%$ yield with moderate to good enantioselectivity (up to $80 \%$ ee).
\end{abstract}

Keywords vinylogous imines; enantioselective conjugate addition; sulfonyl indoles

3-位取代的吲哚衍生物是重要的医药中间体, 在医 药化学中具有重要的作用 ${ }^{[1]}$, 但这类化合物的合成却非

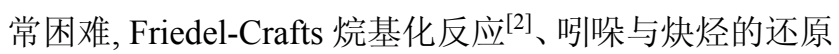
烷基化反应 ${ }^{[3]}$ 是合成 3-位取代吲哚的重要方法. 但在吲 哚 3 位上引入手性烷基基团的方法至今依然非常有限. 其中一个比较直接的办法是吲哚衍生的非官能烯烃的 不对称还原(图 1, Route $\mathrm{A})^{[4]}$, 但该反应需要贵金属如 Ir, $\mathrm{Rh}$ 等, 反应条件也较苛刻, 迄今为止, 没有吲哚衍生的 非官能化烯烃的不对称加成反应的报道. 二乙基锌等金 属有机化合物对原位产生的不饱和亚胺的不对称加成 是合成手性烷基取代的吲哚衍生物的另一个方法(图 1, Route B).

亲核试剂对缺电子共轭不饱和化合物的对映选择 性加成是有机合成化学研究的重要内容 ${ }^{[6]}$, 二乙基锌是 重要的亲核试剂被广泛应用于手性亚磷酰胺一金属络合 物催化的 $\alpha, \beta$-不饱和羰基化合物和亚胺的不对称加成反 应中 ${ }^{[7,8]}$. 磺酰基取代的吲哚衍生物 1 方便易得, 由于苯<smiles>C/C=C(/Br)c1c[nH]c2ccccc12</smiles>

asymmetric reduction<smiles>[R]Oc1c[nH]c2ccccc12</smiles><smiles>COc1ccccc1</smiles>

1<smiles>BrC=c1c2cc1c1ccccc1n2</smiles><smiles>CCC(Br)c1c[nH]c2ccccc12</smiles>

图 1 手性吲哚衍生物的合成路线

Figure 1 Possible routes to chiral indole derivatives

磺酰基在碱的存在下易于离去, 生成不饱和的亚胺化合 物 2, 亲核试剂对中间体 $\mathbf{2}$ 的不对称加成反应能得到光 学活性的吲哚衍生物. 事实上, 丙二腈 ${ }^{[10]}$ 、醛 ${ }^{[11]}$ 、二苯 甲酮亚胺叔丁酯 ${ }^{[12]}$ 、硝基化合物 ${ }^{[13]}$ 、Reformatsk 试剂 ${ }^{[14]}$ 作为亲核试剂, 实现了对亚胺 2 的不对称加成. 尽管如

* E-mail: quanzhongliu@sohu.com

Received July 7, 2012; revised August 2, 2012; published online August 6, 2012.

Project supported by the National Natural Science Foundation of China (Nos. 21102116, 20772097).

国家自然科学基金(Nos. 21102116, 20772097)资助项目。 
此, 金属有机化合物对对原位产生的亚胺 $\mathbf{2}$ 的反应依然 较少 ${ }^{[15]}$, 而二乙基锌对亚胺 $\mathbf{2}$ 的不对称共轭加成还未见 报道, 而该反应的产物是具有药理活性的化合物的重要 片断.

\section{1 结果与讨论}

二乙基锌是强亲核试剂, 同时也是强碱. 我们设想 在二乙基锌的存在下, 磺酰吲哚 $\mathbf{1}$ 将会失去离去基团苯 磺酰基生成 $\alpha, \beta$-不饱和亚胺 2 . 在手性亚磷酰胺一酮络合 物的催化下, 二乙基锌将与原位生成的 $\alpha, \beta$-不饱和亚胺 发生不对称共轭加成. 当磺酰基吲哚衍生物 1 与二乙基 锌在光学纯亚磷酰胺 3a $(4 \mathrm{~mol} \%)$ 与 $\mathrm{Cu}(\mathrm{OTf})_{2}(2 \mathrm{~mol} \%)$ 的络合物存在下 $-20{ }^{\circ} \mathrm{C}$ 发生反应时, 经过 $5 \mathrm{~h}$ 以 $90 \%$ 的 产率得到了相应的加成产物 $\mathbf{4}$, 尽管产物的对映选择性 只有 $27 \% e e$. 我们进一步考察了其他手性亚磷酰胺配 体(图 2)对反应的影响(表 1). 结果显示 $\mathbf{3 g}$ 是最好的配 体，取得了最好的对映选择性和最高的产率(表 1, Entry 7). 配体的立体位阻对反应影响较大, 如 $\mathbf{3 a}$ 和 $\mathbf{3 b}$ 比较, 反应的对映选择性从 $27 \%$ 增加到 32\%, 手性胺的引入如 $3 \mathrm{~g}$ 能极大地增加反应的对映选择性. 进一步考察发现 $S$-构型的联二菜酚与 $R$-构型的手性胺得到的手性亚磷 酰胺配体 $3 \mathrm{~g}$ 是构型匹配的配体.

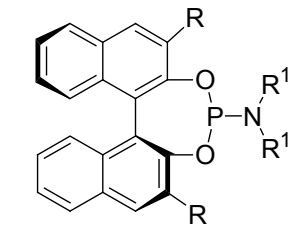

3a $\mathrm{R}=\mathrm{H}, \mathrm{R}^{1}=\mathrm{CH}\left(\mathrm{CH}_{3}\right)_{2}$ 3b $\mathrm{R}=\mathrm{CH}_{3}, \mathrm{R}^{1}=\mathrm{CH}\left(\mathrm{CH}_{3}\right)_{2}$

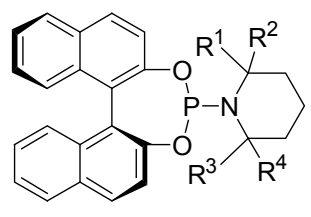

$3 d R^{1}=R^{2}=R^{3}=R^{4}=H$ 3e $\mathrm{R}^{1}=\mathrm{R}^{2}=\mathrm{R}^{3}=\mathrm{R}^{4}=\mathrm{Me}$

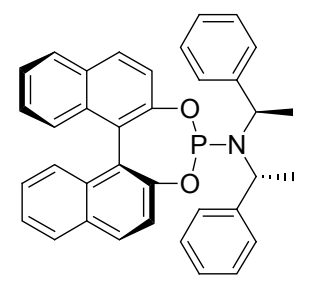

$3 g$

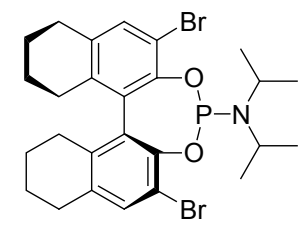

$3 c$

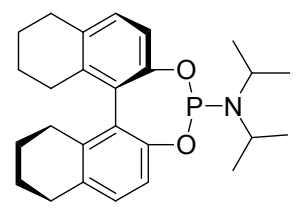

$3 f$
图 2 本文所考察的配体

Figure 1 Chiral phosphoramidites investigated in the work
表 1 二乙基锌对不饱和亚胺的对映选择性加成

Table 1 Enantioselective conjugate addition of vinylogous imines with $\mathrm{Et}_{2} \mathrm{Zn}$

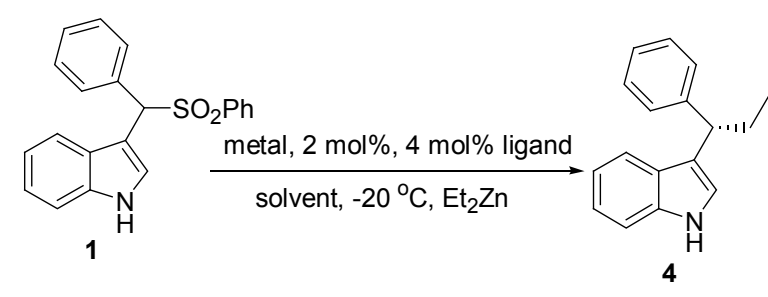

\begin{tabular}{|c|c|c|c|c|c|}
\hline Entry & Ligand & Metal & Solvent & Yield/\% & $e e / \%$ \\
\hline 1 & $3 \mathbf{a}$ & $\mathrm{Cu}(\mathrm{OTf})_{2}$ & Toluene & 90 & 27 \\
\hline 2 & $3 \mathbf{b}$ & $\mathrm{Cu}(\mathrm{OTf})_{2}$ & Toluene & 99 & 32 \\
\hline 3 & $3 c$ & $\mathrm{Cu}(\mathrm{OTf})_{2}$ & Toluene & 83 & 36 \\
\hline 4 & 3d & $\mathrm{Cu}(\mathrm{OTf})_{2}$ & Toluene & 32 & 22 \\
\hline 5 & $3 \mathbf{e}$ & $\mathrm{Cu}(\mathrm{OTf})_{2}$ & Toluene & 73 & 5 \\
\hline 6 & $3 f$ & $\mathrm{Cu}(\mathrm{OTf})_{2}$ & Toluene & 89 & 36 \\
\hline 7 & $3 g$ & $\mathrm{Cu}(\mathrm{OTf})_{2}$ & Toluene & 99 & 73 \\
\hline 8 & $3 h$ & $\mathrm{Cu}(\mathrm{OTf})_{2}$ & Toluene & 55 & 14 \\
\hline 9 & $3 g$ & CuOTf & Toluene & 55 & 46 \\
\hline 10 & $3 g$ & $\mathrm{CuBr}$ & Toluene & 46 & 17 \\
\hline 11 & $3 g$ & $\mathrm{CuI}$ & Toluene & 31 & 63 \\
\hline 12 & $3 g$ & $\mathrm{CuBr}_{2}$ & Toluene & 46 & 18 \\
\hline 13 & $3 g$ & $\mathrm{Cu}(\mathrm{OAc})_{2}$ & Toluene & 21 & 62 \\
\hline 14 & $3 g$ & $\mathrm{CuCl}_{2}$ & Toluene & 25 & 38 \\
\hline 15 & $3 g$ & $\mathrm{Cu}(\mathrm{OTf})_{2}$ & $\mathrm{CH}_{2} \mathrm{Cl}_{2}$ & 93 & 56 \\
\hline 16 & $3 g$ & $\mathrm{Cu}(\mathrm{OTf})_{2}$ & $\mathrm{CHCl}_{3}$ & 56 & 49 \\
\hline 17 & $3 g$ & $\mathrm{Cu}(\mathrm{OTf})_{2}$ & THF & 82 & 54 \\
\hline 18 & $3 g$ & $\mathrm{Cu}(\mathrm{OTf})_{2}$ & $\mathrm{Et}_{2} \mathrm{O}$ & 45 & 59 \\
\hline 19 & $3 g$ & $\mathrm{Cu}(\mathrm{OTf})_{2}$ & CPME & 76 & 52 \\
\hline 20 & $3 g$ & $\mathrm{Cu}(\mathrm{OTf})_{2}$ & $\mathrm{CH}_{3} \mathrm{CN}$ & $<10$ & - \\
\hline $21^{a}$ & $3 g$ & $\mathrm{Cu}(\mathrm{OTf})_{2}$ & Toluene & 99 & 73 \\
\hline $21^{b}$ & $3 g$ & $\mathrm{Cu}(\mathrm{OTf})_{2}$ & Toluene & 83 & 70 \\
\hline $22^{c}$ & $3 \mathrm{~g}$ & $\mathrm{Cu}(\mathrm{OTf})_{2}$ & Toluene & 30 & 63 \\
\hline
\end{tabular}

通过考察金属对反应的影响发现 $\mathrm{Cu}(\mathrm{OTf})_{2}$ 较其他 金属具有良好的催化性能，取得了最高的产率和 $73 \%$ 的 对映选择性. 在尝试的其他铜盐如 $\mathrm{CuCl}_{2}, \mathrm{CuBr}_{2}, \mathrm{CuI}$, $\mathrm{Cu}(\mathrm{OAc})_{2}$ 中, 反应的规律性不很明显, $\mathrm{CuI}$ 和 $\mathrm{Cu}(\mathrm{OAc})_{2}$ 得到的对映选择性要好于其他铜盐. 溶剂笁选发现甲苯 是最好的溶剂, 总的来说, 非极性溶剂有利于反应的进 行，如二氯甲烷和三氯甲烷相比对映选择性从 $56 \%$ 降到 $49 \%$. 其他醚类溶剂也反应出了这一趋势，这也许是极 性强的溶剂易于和 Lewis 酸络合从而降低催化活性从而 产率相应降低. 如二氯甲烷作溶剂反应产率达到 $93 \%$, 而氯仿作反应溶剂时产率降到 56\%; 同样 THF, CPME, $\mathrm{Et}_{2} \mathrm{O}$ 分别作为反应的溶剂时，产率分别为 $82 \%, 76 \%$ 和 $45 \%$. 而当乙腈为反应溶剂时, 反应不能发生. 温度降 低无论是反应的产率和对映选择性均下降, 如在一 20 , -40 和 $-60{ }^{\circ} \mathrm{C}$ 产率从 $99 \%$ 降到 $30 \%$, 而对映选择性没 有明显降低. 
通过条件筛选, 反应的最优条件优化为: 相对于磺 酰吲哚 $2 \mathrm{~mol} \% \mathrm{Cu}(\mathrm{OTf})_{2}$ 和 $4 \mathrm{~mol} \%$ 的手性配体 $\mathbf{3 g}$ 在甲 苯中搅拌一段时间后冷却到 $-20{ }^{\circ} \mathrm{C}$, 加入磺酰吲哚和 磺酰吲哚 2 equiv.的二乙基锌反应. 在此条件下我们对 反应的适用范围进行了扩展, 所得结果列于表 2 中. 各 种不同的磺酰吲哚在手性亚磷酰胺配体 3g-铜络合物存 在下与二乙基锌反应以好的产率和最高 $80 \%$ 的对映选 择性生成相应的光学活性的吲哚衍生物. 总的来说, 富 电子底物比如表 1 中, Entries 1 4 所列的底物相对缺电 子底物取得较好的结果.

表 2 二乙基锌对不饱和亚胺的对映选择性加成的适用范围

Table 2 Scope of substrates of enantioselective conjugate addition of vinylogous imines with $\mathrm{Et}_{2} \mathrm{Zn}$

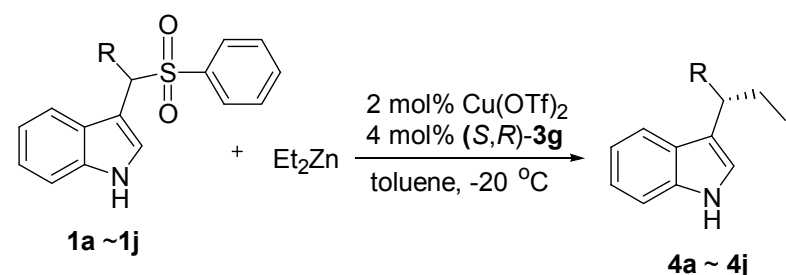

\begin{tabular}{clccc}
\hline Entry & \multicolumn{1}{c}{$\mathrm{R}$} & Product & Yield/\% & $e e / \%$ \\
\hline 1 & $\mathrm{C}_{6} \mathrm{H}_{5}$ & $\mathbf{4 a}$ & 99 & 73 \\
2 & $4-\mathrm{MeC}_{6} \mathrm{H}_{4}$ & $\mathbf{4 b}$ & 85 & 80 \\
3 & $3-\mathrm{MeC}_{6} \mathrm{H}_{4}$ & $\mathbf{4 c}$ & 94 & 68 \\
4 & $2-\mathrm{MeC}_{6} \mathrm{H}_{4}$ & $\mathbf{4 d}$ & 92 & 72 \\
5 & $3-\mathrm{MeOC}_{6} \mathrm{H}_{4}$ & $\mathbf{4 e}$ & 92 & 67 \\
6 & $4-\mathrm{ClC}_{6} \mathrm{H}_{4}$ & $\mathbf{4 f}$ & 85 & 72 \\
7 & $3-\mathrm{ClC}_{6} \mathrm{H}_{4}$ & $\mathbf{4 g}$ & 90 & 46 \\
8 & $4-\mathrm{BrC}_{6} \mathrm{H}_{4}$ & $\mathbf{4 h}$ & 92 & 71 \\
9 & $4-\mathrm{NCC}_{6} \mathrm{H}_{4}$ & $\mathbf{4 i}$ & 46 & 16 \\
10 & Naphthyl & $\mathbf{4 j}$ & 66 & 78 \\
\hline
\end{tabular}

除非特别说明, 所有反应均为 $0.1 \mathrm{mmol}$ 规模, 催 化剂由 $\mathrm{Cu}(\mathrm{OTf})_{2}(2 \mathrm{mmol} \%)$, 手性亚磷酰胺 (4 mmol\%) 搅拌制得并直接使用. 对映选择性由高效液相色谱决 定.

某些光学活性的 3-位烷基吲哚衍生物在正已烷和 乙酸乙酯混合溶剂中简单重结晶能进一步提高其 $e e$ 值, 如 $4 \mathbf{f}$ 重结晶后其光学纯度达到 $99 \%$ ee 以上. 通过对光 学纯 $\mathbf{4 f}$ 的单晶 $\mathrm{X}$ 射线衍射分析, 确定其绝对构型为 $S$ 构型, 其他产物的绝对构型通过类推得到(图 3).

\section{2 结论}

我们发现了二乙基锌对原位产生亚胺的对映选择 性共轭加成反应, 以高达 $99 \%$ 的产率和 $80 \%$ 的对映选择 性得到了光学活性的 3-位烷基取代的吲哚衍生物, 部分 产物通过简单重结晶能得到 $>99 \%$ 的光学纯度, 并通过 单晶确定了产物的绝对构型.

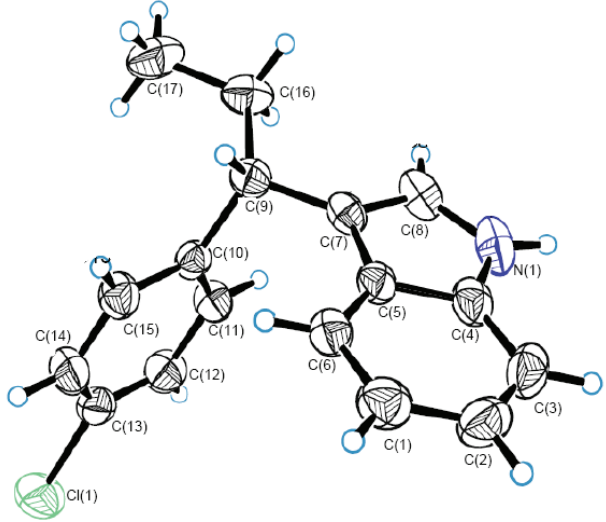

图 3 产物 $\mathbf{4 f}$ 的 $\mathrm{X}$ 射线结构

Figure 3 X-ray structure of $\mathbf{4 f}$

\section{3 实验部分}

\section{1 仪器与试剂}

除非特别说明, 所有试剂均从试剂公司购买直接适 用，柱层析使用 $200 \sim 300$ 目的硅胶. 氢谱和碳谱均 Bruker Avance 400MHz 核磁共振波谱仪测定得到, 手性 高效液相色谱仪由 Shimadzu LC20A 测量(手性柱为 Chirapak OD-H and AD-H columns, Daicel Chemical Ind., Ltd.), 高分辨质谱仪由 Bruker micrOTOF-Q 测定. 手性 配体由文献[16]方法合成, 本文所用到的磺酰吲哚均是 已知化合物并由相应的文献 ${ }^{[10]}$ 合成.

\section{2 实验方法}

$\mathrm{Cu}(\mathrm{OTf})_{2}(0.002 \mathrm{mmol}, 0.8 \mathrm{mg})$, 手性亚磷酰胺配体 $(S, R)$-3g $(0.004 \mathrm{mmol}, 2.2 \mathrm{mg})$ 溶于甲苯 $(1.0 \mathrm{~mL})$ 中并在 氮气氛中室温下搅拌 $1 \mathrm{~h}$, 混合液冷却到 $-20{ }^{\circ} \mathrm{C}$, 加入 磺酰吲哚 1a $(0.1 \mathrm{mmol}, 34.7 \mathrm{mg})$ 溶于甲苯 $(1.0 \mathrm{~mL})$ 的溶 液、 $\mathrm{ZnEt}_{2}$ (0.2 mmol, $0.2 \mathrm{~mL} 1.0 \mathrm{~mol} / \mathrm{L}$ 正已烷溶液). 搅 拌反应 $5 \mathrm{~h}$ 后, 用饱和 $\mathrm{NH}_{4} \mathrm{Cl}(5.0 \mathrm{~mL})$ 淬灭反应，以乙酸 乙酯 $(5.0 \mathrm{~mL} \times 3)$ 萃取, 合并萃取液并用饱和 $\mathrm{NaCl}$ 溶液 $(5.0 \mathrm{~mL} \times 3)$ 洗涤, 以无水 $\mathrm{Na}_{2} \mathrm{SO}_{4}$ 干燥. 溶剂减压除去 后经硅胶柱层析(PE-EtOAc, $V: V=100: 1)$ 得到相应的 加成产物 4.

(S)-3-(1-苯基丙基)- $1 H$-吲哚(4a): 白色固体, 产率 99\%. m.p. $111 \sim 112{ }^{\circ} \mathrm{C},[\alpha]_{\mathrm{D}}^{25}-3.9\left(\mathrm{CHCl}_{3}, c 0.31\right), 73 \% e e$, HPLC 分析(chiral OD-H column, 10\% IPA in hexane, rate: $1.0 \mathrm{~mL} / \mathrm{min}, 254 \mathrm{~nm}$ ); Retention time: $t$ (minor) $=8.4$ min, $t$ (major) $=9.6 \mathrm{~min} ;{ }^{1} \mathrm{H}$ NMR $\left(400 \mathrm{MHz}, \mathrm{CDCl}_{3}\right) \delta$ : $0.96(\mathrm{t}, J=7.4 \mathrm{~Hz}, 3 \mathrm{H}), 1.99 \sim 2.10(\mathrm{~m}, 1 \mathrm{H}), 2.19 \sim 2.30$ $(\mathrm{m}, 1 \mathrm{H}), 4.07(\mathrm{t}, J=7.6 \mathrm{~Hz}, 1 \mathrm{H}), 7.00 \sim 7.04(\mathrm{~m}, 1 \mathrm{H}), 7.05$ (dd, $J=2.4,0.8 \mathrm{~Hz}, 1 \mathrm{H}), 7.13 \sim 7.19(\mathrm{~m}, 2 \mathrm{H}), 7.29 \sim 7.35$ (m, 5H), 7.44 7.46 (m, 1H), 7.97 (bs, $1 \mathrm{H}) ;{ }^{13} \mathrm{C}$ NMR $\left(100 \mathrm{MHz}, \mathrm{CDCl}_{3}\right) \delta: 145.2,136.4,128.1,127.9,127.1$, 
125.8, 121.8, 120.8, 120.4, 119.5, 119.1, 110.9, 44.7, 29.0, 12.7. HRMS calcd for $\mathrm{C}_{17} \mathrm{H}_{18} \mathrm{~N}\left(\mathrm{M}^{+}+\mathrm{H}\right): 236.1439$, found 236.1450 .

(S)-3-(1-对甲基苯基丙基)- $1 H$-吲哚(4b): 白色固体，产 率 $85 \%$, m.p. $69 \sim 70{ }^{\circ} \mathrm{C},[\alpha]_{\mathrm{D}}^{25}-11.1\left(\mathrm{CHCl}_{3}, c 0.21\right)$, $80 \%$ ee, HPLC 分析(chiral OD-H column, 10\% IPA in hexane, rate: $1.0 \mathrm{~mL} / \mathrm{min}, 254 \mathrm{~nm})$; Retention time: $t$ (minor $)=7.7 \mathrm{~min}, t$ (major $)=8.7 \mathrm{~min} ;{ }^{1} \mathrm{H} \mathrm{NMR}(400$ $\left.\mathrm{MHz}, \mathrm{CDCl}_{3}\right) \delta: 0.98(\mathrm{t}, J=7.3 \mathrm{~Hz}, 3 \mathrm{H}), 2.00 \sim 2.10(\mathrm{~m}$, $1 \mathrm{H}), 2.19 \sim 2.30(\mathrm{~m}, 1 \mathrm{H}), 2.33(\mathrm{~s}, 3 \mathrm{H}), 4.06(\mathrm{t}, J=7.6 \mathrm{~Hz}$, $1 \mathrm{H}), 7.02 \sim 7.06(\mathrm{~m}, 2 \mathrm{H}), 7.09 \sim 7.11(\mathrm{~m}, 2 \mathrm{H}), 7.14 \sim 7.18$ $(\mathrm{m}, 1 \mathrm{H}), 7.20 \sim 7.23(\mathrm{~m}, 2 \mathrm{H}), 7.32 \sim 7.35(\mathrm{~m}, 1 \mathrm{H}), 7.47 \sim$ $7.50(\mathrm{~m}, 1 \mathrm{H}), 7.92$ (bs, $1 \mathrm{H}) ;{ }^{13} \mathrm{C} \mathrm{NMR}\left(100 \mathrm{MHz}, \mathrm{CDCl}_{3}\right)$ $\delta: 142.2,136.4,135.2,128.8,127.8,127.1,121.8,120.8$, $120.6,119.5,119.1,110.9,44.3,29.0,20.9,12.8$. HRMS calcd for $\mathrm{C}_{18} \mathrm{H}_{20} \mathrm{~N}\left(\mathrm{M}^{+}+\mathrm{H}\right): 250.1596$, found 250.1589 .

(S)-3-(1-间甲基苯基丙基)- $1 H$-吲哚(4c)：无色粘稠液 体, 产率 94\%, $[\alpha]_{\mathrm{D}}^{25}-4.2\left(\mathrm{CHCl}_{3}, c \quad 0.19\right), 68 \%$ ee, HPLC 分析(chiral OD-H column, 5\% IPA in hexane, rate: $1.0 \mathrm{~mL} / \mathrm{min}, 254 \mathrm{~nm}$ ); Retention time: $t$ (minor) $=11.0 \mathrm{~min}$, $t$ (major) $=13.1 \mathrm{~min} ;{ }^{1} \mathrm{H}$ NMR $\left(400 \mathrm{MHz}, \mathrm{CDCl}_{3}\right) \delta: 0.99$ $(\mathrm{t}, J=7.3 \mathrm{~Hz}, 3 \mathrm{H}), 2.00 \sim 2.11(\mathrm{~m}, 1 \mathrm{H}), 2.18 \sim 2.30(\mathrm{~m}$, $1 \mathrm{H}), 2.34(\mathrm{~s}, 3 \mathrm{H}), 4.06(\mathrm{t}, J=7.6 \mathrm{~Hz}, 1 \mathrm{H}), 7.00 \sim 7.08(\mathrm{~m}$, $3 \mathrm{H}), 7.14 \sim 7.21(\mathrm{~m}, 4 \mathrm{H}), 7.33 \sim 7.35(\mathrm{~m}, 1 \mathrm{H}), 7.51(\mathrm{~d}, J=$ $8.0 \mathrm{~Hz}, 1 \mathrm{H}), 7.91$ (bs, $1 \mathrm{H}) ;{ }^{13} \mathrm{C} \mathrm{NMR}\left(100 \mathrm{MHz}, \mathrm{CDCl}_{3}\right) \delta$ : $145.2,137.6,136.4,128.7,128.0,127.1,126.6,124.9$, $121.8,120.8,120.5,119.5,119.1,110.9,44.7,29.0,21.5$, 12.8. HRMS calcd for $\mathrm{C}_{18} \mathrm{H}_{20} \mathrm{~N}\left(\mathrm{M}^{+}+\mathrm{H}\right): 250.1596$, found 250.1601 .

(S)-3-(1-邻甲基苯基丙基)- $1 H$-吲哚(4d)：无色粘稠液 体, 产率 $92 \%,[\alpha]_{\mathrm{D}}^{25}-13.2\left(\mathrm{CHCl}_{3}, c \quad 0.22\right), 72 \%$ ee, HPLC 分析(chiral OD-H column, 5\% IPA in hexane, rate: $1.0 \mathrm{~mL} / \mathrm{min}, 254 \mathrm{~nm}$ ); Retention time: $t$ (minor) $=10.6 \mathrm{~min}$, $t$ (major) $=11.6 \mathrm{~min} ;{ }^{1} \mathrm{H} \mathrm{NMR}\left(400 \mathrm{MHz}, \mathrm{CDCl}_{3}\right) \delta: 1.02$ $(\mathrm{t}, J=7.3 \mathrm{~Hz}, 3 \mathrm{H}), 1.99 \sim 2.10(\mathrm{~m}, 1 \mathrm{H}), 2.20 \sim 2.31(\mathrm{~m}$, $1 \mathrm{H}), 2.44(\mathrm{~s}, 3 \mathrm{H}), 4.35(\mathrm{t}, J=7.4 \mathrm{~Hz}, 1 \mathrm{H}), 6.91(\mathrm{dd}, J=$ $2.4,0.8 \mathrm{~Hz}, 1 \mathrm{H}), 7.04 \sim 7.08(\mathrm{~m}, 1 \mathrm{H}), 7.09 \sim 7.20(\mathrm{~m}, 4 \mathrm{H})$, $7.28 \sim 7.30(\mathrm{~m}, 1 \mathrm{H}), 7.33 \sim 7.35(\mathrm{~m}, 1 \mathrm{H}), 7.46 \sim 7.48(\mathrm{~m}$, 1H), $7.90(\mathrm{bs}, 1 \mathrm{H}) ;{ }^{13} \mathrm{C} \mathrm{NMR}\left(100 \mathrm{MHz}, \mathrm{CDCl}_{3}\right) \delta: 143.2$, $136.4,135.9,130.2,127.2,126.8,125.9,125.6,121.8$, $121.4,120.4,119.3,119.1,110.9,39.8,29.0,19.8,12.8$. HRMS calcd for $\mathrm{C}_{18} \mathrm{H}_{20} \mathrm{~N}\left(\mathrm{M}^{+}+\mathrm{H}\right)$ : 250.1596, found 250.1593.

(S)-3-(1-间-甲氧基苯基丙基)- $1 H$-吲槑(4e)：无色粘稠 液体, 产率 $92 \%,[\alpha]_{\mathrm{D}}^{25}-1.6\left(\mathrm{CHCl}_{3}, c 0.30\right), 67 \%$ ee,
HPLC 分析(chiral OD-H column, 10\% IPA in hexane, rate: $1.0 \mathrm{~mL} / \mathrm{min}, 254 \mathrm{~nm}$ ); Retention time: $t$ (minor) $=11.2$ $\min , t$ (major) $=15.0 \mathrm{~min} ;{ }^{1} \mathrm{H}$ NMR $\left(400 \mathrm{MHz}, \mathrm{CDCl}_{3}\right) \delta$ : $0.98(\mathrm{t}, J=7.3 \mathrm{~Hz}, 3 \mathrm{H}), 2.00 \sim 2.10(\mathrm{~m}, 1 \mathrm{H}), 2.19 \sim 2.29$ $(\mathrm{m}, 1 \mathrm{H}), 3.78(\mathrm{~s}, 3 \mathrm{H}), 4.06(\mathrm{t}, J=7.7 \mathrm{~Hz}, 1 \mathrm{H}), 6.72 \sim 6.75$ $(\mathrm{m}, 1 \mathrm{H}), 6.88 \sim 6.95(\mathrm{~m}, 2 \mathrm{H}), 7.02 \sim 7.06(\mathrm{~m}, 2 \mathrm{H}), 7.14 \sim$ $7.23(\mathrm{~m}, 2 \mathrm{H}), 7.33$ (d, $J=8.1 \mathrm{~Hz}, 1 \mathrm{H}), 7.50$ (d, $J=8.0 \mathrm{~Hz}$, 1H), 7.94 (bs, $1 \mathrm{H}) ;{ }^{13} \mathrm{C} \mathrm{NMR}\left(100 \mathrm{MHz}, \mathrm{CDCl}_{3}\right) \delta: 159.5$, $147.0,136.4,129.0,127.1,121.8,120.8,120.5,120.3$, 119.4, 119.1, 114.0, 110.9, 110.7, 55.0, 44.8, 28.9, 12.8. HRMS calcd for $\mathrm{C}_{18} \mathrm{H}_{20} \mathrm{NO}\left(\mathrm{M}^{+}+\mathrm{H}\right): 266.1545$, found 266.1541 .

(S)-3-(1-对-氯苯基丙基)- $1 H$-吲哚(4f): 白色固体，产 率 85\%, m.p. 93 $95{ }^{\circ} \mathrm{C},[\alpha]_{\mathrm{D}}^{25}-13.6\left(\mathrm{CHCl}_{3}, c 0.21\right)$, $72 \% e e, \mathrm{HPLC}$ 分析(chiral AD-H column, 10\% IPA in hexane, rate: $1.0 \mathrm{~mL} / \mathrm{min}, 254 \mathrm{~nm})$. Retention time: $t$ (minor) $=9.8 \mathrm{~min}, t$ (major $)=11.2 \mathrm{~min} ;{ }^{1} \mathrm{H}$ NMR $(400$ $\left.\mathrm{MHz}, \mathrm{CDCl}_{3}\right) \delta: 0.97(\mathrm{t}, J=7.3 \mathrm{~Hz}, 3 \mathrm{H}), 1.95 \sim 2.06(\mathrm{~m}$, $1 \mathrm{H}), 2.19 \sim 2.30(\mathrm{~m}, 1 \mathrm{H}), 4.06(\mathrm{dd}, J=7.9,7.2 \mathrm{~Hz}, 1 \mathrm{H})$, $7.02 \sim 7.06(\mathrm{~m}, 2 \mathrm{H}), 7.15 \sim 7.19(\mathrm{~m}, 1 \mathrm{H}), 7.24(\mathrm{~s}, 4 \mathrm{H})$, $7.34 \sim 7.36(\mathrm{~m}, 1 \mathrm{H}), 7.40 \sim 7.42(\mathrm{~m}, 1 \mathrm{H}), 7.97(\mathrm{bs}, 1 \mathrm{H})$; ${ }^{13} \mathrm{C}$ NMR $\left(100 \mathrm{MHz}, \mathrm{CDCl}_{3}\right) \delta: 143.8,136.4,131.4,129.3$, $128.2,126.8,122.0,120.8,119.9,119.3,119.2,111.0$, 44.1, 28.8, 12.6. HRMS calcd for $\mathrm{C}_{17} \mathrm{H}_{17} \mathrm{ClN}\left(\mathrm{M}^{+}+\mathrm{H}\right)$ : 270.1050 , found 270.1043 .

(S)-3-(1-间-氯苯基丙基)- $1 H$-吲哚(4g)：无色粘稠液体， 产率 $90 \%,[\alpha]_{\mathrm{D}}^{25}-9.0\left(\mathrm{CHCl}_{3}, c 0.17\right), 46 \% e e, \mathrm{HPLC}$ 分 析(chiral OD-H column, 10\% IPA in hexane, rate: 1.0 $\mathrm{mL} / \mathrm{min}, 254 \mathrm{~nm}$ ); Retention time: $t$ (minor) $=10.0 \mathrm{~min}$, $t$ (major) $=11.6 \mathrm{~min} ;{ }^{1} \mathrm{H} \mathrm{NMR}\left(400 \mathrm{MHz}, \mathrm{CDCl}_{3}\right) \delta: 0.99$ $(\mathrm{t}, J=7.3 \mathrm{~Hz}, 3 \mathrm{H}), 1.99 \sim 2.10(\mathrm{~m}, 1 \mathrm{H}), 2.20 \sim 2.30(\mathrm{~m}$, 1H), 4.07 (t, $J=7.7 \mathrm{~Hz}, 1 \mathrm{H}), 7.05 \sim 7.09(\mathrm{~m}, 2 \mathrm{H}), 7.16 \sim$ $7.23(\mathrm{~m}, 4 \mathrm{H}), 7.31 \sim 7.37(\mathrm{~m}, 2 \mathrm{H}), 7.45 \sim 7.47(\mathrm{~m}, 1 \mathrm{H})$, 7.95 (bs, $1 \mathrm{H}) ;{ }^{13} \mathrm{C}$ NMR (100 $\left.\mathrm{MHz}, \mathrm{CDCl}_{3}\right) \delta: 147.5$, $136.4,134.0,129.4,128.0,126.9,126.2,126.1,122.0$, 120.9, 119.6, 119.3, 119.3, 111.0, 44.5, 28.9, 12.7. HRMS calcd for $\mathrm{C}_{17} \mathrm{H}_{17} \mathrm{ClN}\left(\mathrm{M}^{+}+\mathrm{H}\right)$ : 270.1050, found 270.1045 .

(S)-3-(1-对-溴苯基丙基)- $1 H$-吲哚(4h): 白色固体, 产率 $92 \%$, m.p. $84 \sim 85{ }^{\circ} \mathrm{C},[\alpha]_{\mathrm{D}}^{25}-15.0\left(\mathrm{CHCl}_{3}, c \quad 0.27\right), 71 \%$ $e e$, HPLC 分析(chiral AD-H column, 10\% IPA in hexane, rate: $1.0 \mathrm{~mL} / \mathrm{min}, 254 \mathrm{~nm}$ ). Retention time: $t$ (minor) $=10.3$ min, $t$ (major) $=11.9 \mathrm{~min} ;{ }^{1} \mathrm{H}$ NMR $\left(400 \mathrm{MHz} \mathrm{CDCl}_{3}\right) \delta$ : $0.97(\mathrm{t}, J=7.3 \mathrm{~Hz}, 3 \mathrm{H}), 1.95 \sim 2.06(\mathrm{~m}, 1 \mathrm{H}), 2.19 \sim 2.30$ $(\mathrm{m}, 1 \mathrm{H}), 4.05(\mathrm{t}, J=7.6 \mathrm{~Hz}, 1 \mathrm{H}), 7.03 \sim 7.07(\mathrm{~m}, 2 \mathrm{H})$, $7.16 \sim 7.21(\mathrm{~m}, 3 \mathrm{H}), 7.33 \sim 7.36(\mathrm{~m}, 1 \mathrm{H}), 7.38 \sim 7.42(\mathrm{~m}$, 
3H), 7.96 (bs, $1 \mathrm{H}) ;{ }^{13} \mathrm{C} \mathrm{NMR}\left(100 \mathrm{MHz}, \mathrm{CDCl}_{3}\right) \delta: 144.3$, $136.4,131.2$, 129.7, 126.8, 122.0, 120.9, 119.8, 119.5, 119.3, 119.2, 111.0, 44.2, 28.8, 12.6; HRMS calcd for $\mathrm{C}_{17} \mathrm{H}_{17} \mathrm{BrN}\left(\mathrm{M}^{+}+\mathrm{H}\right): 314.0544$, found 314.0538 .

(S)-3-(1-对氰基苯基丙基)- $1 H$-吲哚(4i): 白色固体，产 率 46\%, m.p. $135 \sim 136{ }^{\circ} \mathrm{C},[\alpha]_{\mathrm{D}}^{25}+5.5\left(\mathrm{CHCl}_{3}, c 0.12\right)$, $16 \%$ ee, HPLC 分析 (chiral AD-H column, 5\% IPA in hexane, rate: $1.0 \mathrm{~mL} / \mathrm{min}, 254 \mathrm{~nm})$; Retention time: $t$ (minor $)=60.3 \mathrm{~min}, t$ (major $)=64.6 \mathrm{~min} ;{ }^{1} \mathrm{H} \mathrm{NMR}(400$ $\left.\mathrm{MHz}, \mathrm{CDCl}_{3}\right) \delta: 0.96(\mathrm{t}, J=7.3 \mathrm{~Hz}, 3 \mathrm{H}), 1.97 \sim 2.08(\mathrm{~m}$, $1 \mathrm{H}), 2.20 \sim 2.31(\mathrm{~m}, 1 \mathrm{H}), 4.12(\mathrm{t}, J=7.6 \mathrm{~Hz}, 1 \mathrm{H}), 7.00 \sim$ $7.04(\mathrm{~m}, 1 \mathrm{H}), 7.09(\mathrm{~d}, J=2.4 \mathrm{~Hz}, 1 \mathrm{H}), 7.15 \sim 7.19(\mathrm{~m}$, $1 \mathrm{H}), 7.32 \sim 7.41(\mathrm{~m}, 4 \mathrm{H}), 7.54 \sim 7.57(\mathrm{~m}, 2 \mathrm{H}), 8.07$ (bs, $1 \mathrm{H}) ;{ }^{13} \mathrm{C}$ NMR (100 MHz, $\left.\mathrm{CDCl}_{3}\right) \delta: 151.1,136.4,132.1$, $128.7,126.6,122.2,121.0,119.4,119.1,119.0,118.7$, 111.1, 109.6, 44.8, 28.6, 12.6. HRMS calcd for $\mathrm{C}_{18} \mathrm{H}_{17} \mathrm{~N}_{2}$ $\left(\mathrm{M}^{+}+\mathrm{H}\right): 266.1392$, found 266.1385 .

(S)-3-(1- $\alpha$-萘基丙基)- $1 H$-吲哚(4j): 无色粘稠液体，产 率 $66 \%,[\alpha]_{\mathrm{D}}^{25}+31.4\left(\mathrm{CHCl}_{3}, c 0.11\right), 78 \% e e, \mathrm{HPLC}$ 分 析(chiral OD-H column, 5\% IPA in hexane, rate: 1.0 $\mathrm{mL} / \mathrm{min}, 254 \mathrm{~nm}$ ); Retention time: $t$ (minor) $=15.7 \mathrm{~min}$, $t$ (major) $=17.6 \mathrm{~min} ;{ }^{1} \mathrm{H} \mathrm{NMR}\left(400 \mathrm{MHz}, \mathrm{CDCl}_{3}\right) \delta: 1.06$ $(\mathrm{t}, J=7.3 \mathrm{~Hz}, 3 \mathrm{H}), 2.18 \sim 2.29(\mathrm{~m}, 1 \mathrm{H}), 2.30 \sim 2.41(\mathrm{~m}$, $1 \mathrm{H}), 4.97$ (t, $J=7.3 \mathrm{~Hz}, 1 \mathrm{H}), 6.97$ (dd, $J=2.4,0.8 \mathrm{~Hz}$, $1 \mathrm{H}), 6.99 \sim 7.03(\mathrm{~m}, 1 \mathrm{H}), 7.14 \sim 7.18(\mathrm{~m}, 1 \mathrm{H}), 7.33 \sim 7.35$ $(\mathrm{m}, 1 \mathrm{H}), 7.38 \sim 7.51(\mathrm{~m}, 5 \mathrm{H}), 7.72(\mathrm{~d}, J=7.8 \mathrm{~Hz}, 1 \mathrm{H})$, $7.85 \sim 7.89$ (m, 1H), 7.93 (bs, 1H), 8.26 8.29 (m, 1H); ${ }^{13} \mathrm{C} \mathrm{NMR}\left(100 \mathrm{MHz}, \mathrm{CDCl}_{3}\right) \delta: 141.0,136.4,133.9,132.0$, $128.8,127.2,126.5,125.6,125.4,125.1,124.3,123.5$, $121.8,121.7,120.4,119.4,119.1,110.9,39.3,29.1,13.0$. HRMS calcd for $\mathrm{C}_{21} \mathrm{H}_{20} \mathrm{~N}\left(\mathrm{M}^{+}+\mathrm{H}\right)$ : 286.1596, found 286.1587 .

\section{References}

[1] Mannix, L. K.; Files, J. A. CNS Drugs 2005, 19, 951.

(b) Goadsby, P. J. Nat. Rev. Drug Discovery 2005, 4, 741.

(c) Buchanan, T. M.; Ramadan, N. M.; Aurora, S. Exp. Rev. Neurother. 2004, 4, 391.

[2] (a) Trofimov, B. A.; Nedolya, N. A. In Comprehensive Heterocyclic Chemistry III, Vol. 3, Eds.: Katritzky, A. R.; Ramsden, C. A.; Scriven, E. F. V.; Taylor, R. J. K.; Jones, G.; Elsevier, Oxford, 2008, pp. $110 \sim 133$.

(b) Bandini, M.; Eichholzer, A. Angew. Chem., Int. Ed. 2009, 48, 9608 .

(c) Rosa, M. D.; Soriente, A. Eur. J. Org. Chem. 2010, 1029.

(d) Desroses, M.; Wieckowski, K.; Stevens, M.; Odell, L. R. Tetrahedron Lett. 2011, 52, 4417.

(e) Ballini, R.; Palmieri, A.; Petrini, M.; Torregiani, E. Org. Lett. 2006, 8, 4093.

[3] (a) Trsushimoto, T.; Kanbara, M. Org. Lett. 2011, 13, 912. (b) Rizzo, J. R.; Alt, C. A.; Zhang, T. Y. Tetrahedron Lett. 2008 49, 6749 .

(c) Imm, S.; Bähn, S.; Tillack, A.; Mevius, K.; Nenbert, L.; Beller, M. Chem. Eur. J. 2010, 16, 2705.

[4] (a) Noyori, R. Asymmetric Catalysis in Organic Synthesis, Wiley, New York, 1994

(b) Ojima, I. Catalytic Asymmetric Synthesis, Wiley-VCH, New York, 2000.

(c) Cornils, B.; Herrmann, W. A. Applied Homogeneous Catalysis with Organimetallic Compounds, 2nd ed., Wiley-VCH, Weinheim, 2002.

(d) Woodmansee, D. H.; Pfaltz, A. In Topics in Organometallic Chemistry, Springer-Verlag BerlinHeidelberg, 2011, pp. 31 76.

[5] (a) Cui, X.; Burgess, K. Chem. Rev. 2005, 105, 3272.

(b) Roseblade, S. J.; Pfaltz, A. Acc. Chem. Res. 2007, 40, 1402.

(c) Perry, M. C.; Cui, X.; Powell, M. T.; Hou, D. R.; Reibenspies, J. H.; Burgess, K. J. Am. Chem. Soc. 2003, 125, 113.

(d) Cui, X.; Burgess, K. J. Am. Chem. Soc. 2003, 125, 14212.

(e) Zhao, J.; Burgess, K. J. Am. Chem. Soc. 2009, 131, 13236.

(f) Mazuela, J.; Norrby, P.-O.; Andersson, P. G.; Pàmies, O.; Diéguez, M. J. Am. Chem. Soc. 2011, 133, 13634.

[6] (a) Pelmutter, P. Conjugate Addition Reactions in Organic Synthesis, Pergamon, Oxford, 1992.

(b) Jacobsen, E. N.; Pfaltz, A.; Yamamoto, H. Comprehensive Asymmetric Catalysis, Vol. III, Springer, New York, 1999, pp. $1105 \sim 1143$.

(c) Berner, M.; Tedeschi, L.; Enders, D. Eur. J. Org. Chem. 2002, 1877.

(d) Ballini, R.; Bosica, G.; Fiorini, D.; Palmieri, A.; Petrini, M. Chem. Rev. 2005, 105, 933.

(e) Sulzer-Mossé, S.; Alexakis, A. Chem. Commun. 2007, 3123. (f) Jautze, S.; Peters, R. Synthesis 2010, 365.

[7] Selected recent reviews about asymmetric conjugate additions: (a) Christoffers, J.; Koripelly, G.; Rosiak, A.; Rössle, M. Synthesis 2007, 1279.

(b) Alexakis, A.; Bäckvall, J. E.; Krause, N.; Pàmies, O.; Diéguez, M. Chem. Rev. 2008, 108, 2796.

(c) Harutyunyan, S. R.; den Hartog, T.; Geurts, K.; Minnaard, A. J.; Feringa, B. L. Chem. Rev. 2008, 108, 2824.

(d) Jautze, S.; Peters, R. Synthesis 2010, 365.

[8] (a) Feringa, B. L. Acc. Chem. Res. 2000, 33, 346.

(b) Krause, N.; Hoffmann-Röder, A. Synthesis 2001, 171.

(c) Alexakis, A.; Benhaim, C. Eur. J. Org. Chem. 2002, 3221.

(d) Li, K.; Alexakis, A. Tetrahedron Lett. 2005, 46, 8019.

(e) Li, K.; Alexakis, A. Tetrahedron Lett. 2005, 46, 5823.

(f) Isleyen, A.; Dogan, Ö. Tetrahedron: Asymmetry 2007, 18, 679.

(g) Escorihuela, J.; Burguete, M. I.; Luis, S. V. Tetrahedron Lett. 2008, 49, 6885 .

(h) Dong, Z.-B.; Liu, B.; Fang, C.; Li, J.-S. J. Organomet. Chem. 2008, 693, 17.

(i) Kawamura, K.; Fukukawa, H.; Hayashi, M. Org. Lett. 2008, 10, 3509 .

(j) Xie, Y.; Huang, H.; Mo, W.; Fan, X.; Shen, Z.; Shen, Z.; Sun, N.; Hu, B.; Hu, X. Tetrahedron: Asymmetry 2009, $20,1425$.

(k) Endo, K.; Ogawa, M.; Shibata, T. Angew. Chem., Int. Ed. 2010, 49, 2410.

(1) Tauchman, J.; Císařová, I.; Štěpnička, P. Eur. J. Org. Chem. 2010, 4276.

(m) Zhao, Q.-L.; Tse, M. K.; Wang, L. L.; Xing, A.-P.; Jiang, X. Tetrahedron: Asymmetry 2010, 21, 2788.

(n) Rachwalski, M.; Leśniak, S.; Kiełbasiński, P. Tetrahedron Asymmetry 2010, 21, 1890. 
(o) Miklášová, N. N.; Julínek, O.; Mešková, M.; Setnička, V.; Urbanová, M. Tetrahderon Lett. 2010, 51, 1966.

(p) Ni, C.-Y.; Kan, S.-S.; Liu, Q.-Z.; Kang, T.-R. Org. Biomol. Chem. 2011, 9, 6211.

[9] Palmieri, A.; Petrini, M.; Shaikh, R. R. Org. Biomol. Chem. 2010, $8,1259$.

[10] Jing, L.; Wei, J.; Zhou. L.; Huang, Z.; Li, Z.; Wu, D.; Xiang, H.; Zhou, X. Chem. Eur. J. 2010, 16,10955.

[11] Shaikh, R. R.; Mazzanti, A.; Petrini, M.; Bartoli, G.; Melchiorre, P. Angew. Chem., Int. Ed. 2008, 47, 8707.

[12] Zheng, B.-H.; Ding, C.-H.; Hou, X.-L; Dai, L.-X. Org. Lett. 2010,
12,1688 .

[13] (a) Dobish, M. C.; Johnston, J. N. Org. Lett. 2010, 12, 5744.

(b) Ballini, R.; Palmieri, A.; Petrini, M.; Shaikh, R. R. Adv. Synth. Catal. 2008, 350, 129.

[14] Palmieri, A.; Petrini, M. J. Org. Chem. 2007, 72, 1863.

[15] Marcili, L.; Palmieri, A.; Petrini, M. Org. Biomol. Chem. 2010, 8, 706.

[16] Jagt, R. B. C.; Toullec, P. Y.; Schudde, E. P.; de Vries, J. G.; Feringa, B. L.; Minnaard, A. J. J. Comb. Chem. 2007, 9, 407.

(Qin, X.) 